\title{
Transcranial magnetic stimulation in the treatment of obsessive-compulsive disorder: current perspectives
}

This article was published in the following Dove Press journal:

Neuropsychiatric Disease and Treatment

\author{
Ana Lusicic' \\ Koen RJ Schruers ${ }^{2}$ \\ Stefano Pallanti ${ }^{3,4}$ \\ David J Castle $\mathrm{e}^{5,6}$
}

'PACE Clinic, Orygen Youth Health, Melbourne, VIC, Australia; ${ }^{2}$ Research Institute for Mental Health and Neuroscience, Maastricht University, Maastricht, the Netherlands; ${ }^{3}$ Institute of Neurosciences, Florence, Italy; ${ }^{4}$ Stanford University, Palo Alto, CA, USA; ${ }^{5}$ St Vincent's Hospital, 'University of Melbourne, Melbourne, VIC, Australia
Correspondence: Ana Lusicic PACE Clinic, Orygen Youth Health, 35 Poplar Road, Parkville, Melbourne, VIC 3052, Australia Email ana.lusicic@mh.org.au

\begin{abstract}
Repetitive transcranial magnetic stimulation (rTMS) is a noninvasive neurostimulation technique receiving increasing attention in the treatment of different psychiatric disorders. Evidence for rTMS use in obsessive-compulsive disorder (OCD) is accumulating and informing further developments in the neurostimulation field, the latest being deep transcranial magnetic stimulation (dTMS). dTMS allows direct stimulation of deeper subcortical structures and larger brain volume than conventional rTMS. Underlying neurobiological mechanisms related to transcranial magnetic stimulation are still under evaluation, but appear to offer a novel "third" way of addressing symptoms via localized electrical stimulation compared to pharmacotherapy and psychotherapy approaches. This systematic review focuses on the effects of rTMS and dTMS stimulation on different brain targets in OCD. Brain areas included are the dorsolateral prefrontal cortex, supplementary motor area, orbitofrontal cortex/medial prefrontal cortex, and anterior cingulate cortex (ACC). Improved understanding of the therapeutic effects of rTMS in OCD will support fine-tuning of the method and help determine how we can best optimize the approach via rTMS or dTMS to achieve clinically relevant results.
\end{abstract}

Keywords: repetitive transcranial magnetic stimulation, rTMS, deep TMS, obsessive-compulsive disorder, OCD, noninvasive neurostimulation

\section{Introduction}

Obsessive-compulsive disorder (OCD), characterized by intrusive obsessive and repetitive compulsive symptoms as per DSM5 and ICD10 diagnostic criteria, ${ }^{1,2}$ is known for its chronic, waxing, and waning course and detrimental effect on quality of life. ${ }^{3}$ At $1.6 \%-2.3 \%$, lifetime prevalence is consistent across cultures. ${ }^{4,5}$ Although there is an initial male predominance in childhood and adolescence, by early adulthood gender distribution is almost equal. ${ }^{6}$ Many patients experience delay in recognition and treatment, ${ }^{7}$ with high rates of patients failing to achieve full symptomatic remission despite therapy. ${ }^{8,9}$

Worldwide, OCD treatment guidelines ${ }^{10-12}$ indicate the effectiveness of both pharmacological and psychological treatments. Consistent first-line recommendations include serotonin-reuptake inhibitors (SRIs), including selective SRIs (SSRIs) or clomipramine, and exposure/response prevention cognitive behavioral therapy strategies. Many (40\%-60\%) patients do not show clinically meaningful response to an SRI, with only a third showing response after switching to another SRI. ${ }^{13,14}$ Other treatment strategies include augmentation with antipsychotics and use of adjunctive treatments, such as exercise, motivational interviewing, ${ }^{10,12,15}$ and cognitive therapy or acceptance and commitment therapy. ${ }^{10}$ However, many patients remain symptomatic and impaired. (c)
hereby accept the Terms. Non-commercial uses of the work are permitted without any further permission from Dove Medical Press Limited, provided the work is properly attributed. For permission for commercial use of this work, please see paragraphs 4.2 and 5 of our Terms (https://www.dovepress.com/terms.php). 
Therefore, other strategies, notably ones that directly impact OCD neurocircuitry, are of longstanding interest.

The neurobiology of OCD is well established, and an understanding of neurocircuitry can help inform treatments, notably brain stimulation and lesion approaches. Neurobiological models of OCD describe alterations in cortico-striatothalamo-cortical (CSTC) circuits responsible for affective, cognitive, and motor functions. More recently, other brain regions, such as the limbic and parietal cortices, have also been implicated. ${ }^{16}$

Historically, Alexander et $\mathrm{al}^{17}$ described five parallel basal ganglia-thalamocortical circuits, comprising specific areas of the cerebral cortex linked with the striatum, pallidum, substantia nigra, and thalamus. Subsequently, it was shown that CSTC loops were interconnected and not as segregated as previously considered. ${ }^{16-19}$ "Net activity" of CSTC circuitry is thought to be modulated by the interplay between "direct" and "indirect" pathways connecting the cortex and subcortical structures. The "direct pathway" is thought to be driven by glutamate and $\gamma$-aminobutyric acid-neurotransmitter interplay among the cortex, striatum, and globus pallidus, resulting in disinhibition of the thalamus and excitation of the cortex. The "indirect pathway", with its $\gamma$-aminobutyric acid-inhibitory projections from the striatum to the globus pallidus externa and subthalamic nucleus, excites the globus pallidus interna and inhibits the thalamic input to the cortex. CTSC loops implicated in OCD include the components outlined in the following sections.

\section{Motor circuit/sensorimotor circuit}

This connects the sensorimotor and motor cortices, including the supplementary motor area (SMA) with putamen. Putamen projects to the globus pallidus interna and externa and caudal substantia nigra. The internal pallidal segment in turn projects to the ventrolateral thalamic nucleus and finally back to the sensorimotor cortex. Functional alteration of the loop might be responsible for interfering with suppression of "internally triggered intrusive and repetitive movements and thoughts". ${ }^{20}$

\section{Dorsal cognitive circuit/dorsolateral prefrontal circuit}

This projects from the dorsal prefrontal region (dorsolateral prefrontal cortex [dlPFC], including the dorsal anterior cingulate cortex $[\mathrm{ACC}])^{21}$ to the dorsolateral caudate nucleus, which is further connected to dorsomedial parts of the globus pallidus and rostral substantia nigra. Functionally, the dlPFC is linked to executive cognitive functions, including adjustment of cognitive and behavioral programs. ${ }^{18,19}$ Deficits in this circuits might be linked to increased attention to obsessive thoughts. ${ }^{22}$

\section{Ventral cognitive circuit}

This is formed by projections from the anterolateral orbitofrontal cortex (OFC) to the ventromedial caudate nucleus, which in turn innervates the dorsomedial part of the internal pallidum and rostromedial substantia nigra. Lesions to the lateral OFC appear to interfere with the ability to make appropriate shifts in behavioral sets and may be relevant for ritualized behavioral responses. ${ }^{16,17}$ Hyperactivity of the lateral OFC in this context may mediate obsessions. ${ }^{16}$ It has been suggested that excessive activity in the direct pathway of the OFC-ACC loop "traps obsessive thoughts". ${ }^{23}$

\section{Affective anterior cingulate circuit}

This connects the ventromedial prefrontal cortex (PFC), medial OFC, and ventral $\mathrm{ACC}^{24}$ with the ventral striatum, the nucleus accumbens, and further the ventral pallidum and substantia nigra. It closes the loop by projection to the thalamus and back to the ACC and ventromedial PFC. It appears to be relevant for processing affective and reward information. In OCD, its hyperactivity is linked to altered monitoring of behavior and impairment in modifying behavioral responses.

Historically, neurosurgery was considered the last treatment option for OCD after pharmacological and psychological strategies had been exhausted. Targets have included the cingulate gyrus and anterior limb of the internal capsule, as well as tracts that connect the cortex to thalamic nuclei. Such interventions have shown variable levels of effectiveness, with variable long-term success. ${ }^{11}$ Also, such interventions are irreversible. More recently, there has been interest in noninvasive neuromodulation, including repetitive transcranial magnetic stimulation (rTMS), transcranial direct-current stimulation, and more recently deep transcranial magnetic stimulation (dTMS). rTMS uses a low-intensity magnetic field applied to the scalp surface via specially designed coils to induce neurophysiological changes in the cortex and subcortical areas. Stimulation protocols include low-frequency (LF) rTMS $(<5 \mathrm{~Hz})$, inducing decreased neuronal activity in the cortex, ${ }^{25}$ or high-frequency (HF) rTMS $(>5 \mathrm{~Hz})$, considered to increase the cortical activity via facilitation of synaptic efficiency. ${ }^{26}$ dTMS uses specifically designed coils to reach deeper subcortical structures while generating a localized magnetic field.

Published systematic reviews and meta-analyses ${ }^{27-31}$ have focused on the overall effectiveness of neurostimulation 
in OCD, with mixed conclusions. Such reviews have not explored the neurobiological rationale of modulation of different brain targets that subsume neurobiological mechanisms driving obsessive, compulsive, and anxiety symptoms in OCD. Extant systematic reviews and meta-analysis of neuromodulation in OCD have not fully explored the heterogeneity of treatment parameters. Therefore, this systematic review analyzes randomized controlled trials (RCTs) of rTMS and dTMS stimulation over different brain targets in OCD and using different stimulation parameters. Targeted brain areas included in the review are the dlPFC, SMA, OFC/medial PFC (mPFC), and ACC, all of which have been implicated in OCD.

\section{Materials and methods}

\section{Search strategy}

Separate searches for randomized sham-controlled trials and systematic reviews (including meta-analyses) were conducted using the PubMed/Medline, Ovid SP, and PsycInfo databases for the period from January 1980 to October 2017, accompanied by a manual search for additional references from identified publications (Figure 1). Search terms were as follows: transcranial magnetic stimulation or transcranial magnetic stimulation (TMS) + obsessive compulsive disorder or OCD. Inclusion criteria for this systematic review were sham-controlled trials in humans using active rTMS, $\theta$-burst rTMS, or dTMS versus Sham TMS, double-blind or singleblind randomized trials, and English language articles. We excluded nonrandomized trials, case series, case reports, and uncontrolled trials. Seven systematic reviews and metaanalyses were identified using the same keywords with systematic reviews and/or a meta-analysis filter.

\section{rTMS of dIPFC}

The dlPFC emerged as a target for OCD treatment after converging evidence accrued via neuroimaging research, noting its relevance in the pathophysiology of CSTC networks, specifically of the dorsal cortical (cognitive/associative) loop. Functionally, the "dorsal cognitive loop" is known to be involved in executive functions of planning and working memory and implementation of control via attention during execution of tasks..$^{19,32,33}$ The dlPFC has also been shown to be relevant for retaining and manipulating spatial information $^{34}$ and integration of emotional and cognitive processing. ${ }^{16,19}$ Involvement of the dorsal cognitive loop in OCD is underpinned by neuroimaging findings involving structural, resting-state, symptom provocation, and cognitive task paradigms and studies exploring functional changes associated with treatment. One proposition in understanding

Records identified by database search + full text articles assessed for eligibility:

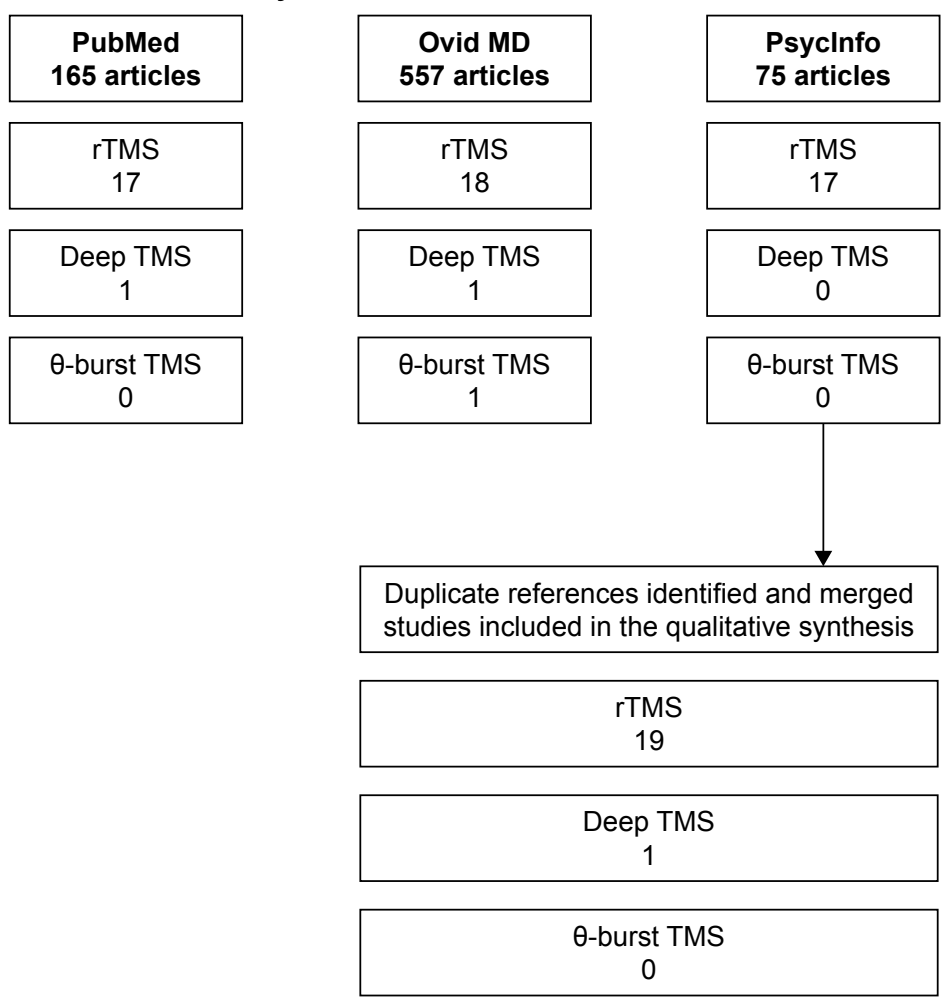

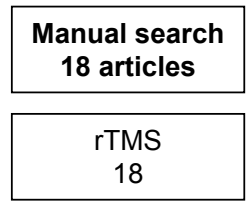
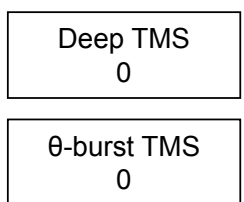

Figure I Search strategy.

Abbreviations: rTMS, repetitive transcranial magnetic stimulation; TMS, transcranial magnetic stimulation. 
the deficits of the dorsal cognitive loop in OCD is dominance of the indirect over direct cortical pathway. ${ }^{19,35}$ The superficial position of the dlPFC, easily accessible to magnetic stimulation, made it an appealing target in the treatment of OCD, especially as it was already shown to be an effective target in the treatment of depression. Greenberg et a ${ }^{36}$ were the first to study the effects of rTMS on the PFC. A single 20-minute session of $20 \mathrm{~Hz}$ rTMS applied over the prefrontal area in 12 OCD patients was associated with a decrease in compulsive symptoms during as well as 30 minutes $(P \leq 0.01)$ and 8 hours after $(P<0.02)$ right PFC stimulation. Left PFC stimulation $(P=0.05)$ did not show this effect.

\section{Studies using LF ( I Hz) rTMS over the right dIPFC}

Several studies have studied LF stimulation over the right dlPFC. Alonso et $\mathrm{al}^{37}$ examined the effects of LF vs sham stimulation over the right dlPFC in a double-blind RCT with 18 unmedicated and medicated OCD subjects. The right dlPFC, inclusive of Brodmann areas (BAs) 6, 8, 10, 44, and 45 , was stimulated using a circular coil with $1 \mathrm{~Hz}$ frequency three times a week for 6 weeks. Baseline mean Yale-Brown Obsessive-Compulsive Scale (YBOCS) scores were in the moderate-severe range. There were no statistically significant effects of active treatment compared to sham treatment on YBOCS $(P=0.81)$ or Hamilton Depression Rating Scale (HDRS) scores $(P=0.95)$ at 10 weeks. The authors raised type II error as a potential explanation for the negative findings. Inclusion of treatment-resistant (TR) patients, heterogeneity in medication status, unclear duration of illness, and active sham coil might have contributed to negative results. Importantly, use of a circular coil has previously been shown to have limited convergence of neurostimuli. ${ }^{38}$ In an RCT of 27 TR OCD patients, Seo et $\mathrm{al}^{39}$ employed 1 $\mathrm{Hz}$ 20-minute train stimulations of the right DLPFC with a figure-eight coil over 3 weeks. The sham protocol was identical, apart from the use of an inactive coil. There was a highly significant difference between two groups after 3 weeks on YBOCS score $(P=0.008)$. However, no significant difference was found in the number of responders to treatment (reduction in YBOCS scores $\geq 25 \%$ ) between groups $(P=0.148)$. Between-group effects were significant for the HDRS $(P=0.009)$ at week 2 and Clinical Global Impression - severity (CGI-S) scale scores at week $3(P=0.008)$. The authors did not address whether the improved YBOCS scores could have been secondary to improved mood. Other limitations included the small sample, lack of double blinding, and potential variability between sessions in precise mapping of targets (Table 1).
In a separate study, Elbeh et $\mathrm{al}^{40}$ examined the effects of $1 \mathrm{~Hz}, 10 \mathrm{~Hz}$, or sham treatment applied to the right dlPFC in 45 OCD patients with a mean duration of illness of $19.2 \pm 11.5$ months. Stimulation was applied over 10 sessions, with a follow-up of 3 months. Sham-stimulation parameters were identical to the $10 \mathrm{~Hz}$ group, but the coil was set at $90^{\circ}$ to the scalp. There was a statistically significant difference between the $1 \mathrm{~Hz}$ rTMS group and the sham group on YBOCS scores at 2 weeks and 3 months $(P=0.001)$, but no significant difference on YBOCS scores for the $10 \mathrm{~Hz}$ group vs the sham group at 2 weeks $(P=0.08)$ or 3 months $(P=0.098)$. The group receiving $1 \mathrm{~Hz}$ frequency showed a statistically significant reduction in YBOCS scores compared to the $10 \mathrm{~Hz}$ group $(P=0.019)$ after 3 months. The study showed good response to $1 \mathrm{~Hz}$ stimulation in young, non-TR OCD patients with short mean duration of illness compared to the $10 \mathrm{~Hz}$ and sham groups. Interestingly, Hamilton Anxiety Rating Scale (HARS) scores reduced significantly in the $1 \mathrm{~Hz}$ group in comparison with the $10 \mathrm{~Hz}$ and sham treatment, but were not addressed as potentially relevant to the decrease in YBOCS scores (Table 1).

\section{Studies using HF ( $\geq 5 \mathrm{~Hz}$ ) rTMS over the right dIPFC} The effects of $10 \mathrm{~Hz}$ rTMS over the right dlPFC were further tested in a 6-week double-blind RCT, followed by a 6-week open-label phase in 27 TR OCD patients with baseline YBOCS scores in the severe-extreme range. ${ }^{41}$ There were no significant differences between groups after 6 weeks of initial treatment $(P=0.710)$. Between-group differences on HDRS $(P=0.637)$ and HARS $(P=0.539)$ scores were not significant either. The additional 6 weeks of open-label active rTMS in 10 patients from the sham group significantly reduced YBOCS scores by week $8(P=0.023)$, with a rebound thereafter. There were no clinical responders in the active group (defined as $\geq 30 \%$ improvement in YBOCS with "much improved/very much improved" CGI scores). Long duration and severity of illness might have contributed to the modest effects of treatment (Table 1).

\section{Studies using LF (I Hz) rTMS over the left dIPFC}

In a double-blind RCT study with 33 TR OCD patients, $1 \mathrm{~Hz}$ or sham stimulation was applied over the left dlPFC for 2 weeks. ${ }^{42}$ Active and sham stimulation was positioned at $90^{\circ}$ over the same area with the same stimulation parameters as the active coil. There were no statistically significant differences between active and sham treatments at 2 and 4 weeks poststimulation. There was a significant difference between baseline YBOCS scores in the active (YBOCS 29.82 \pm 5.8 ) 


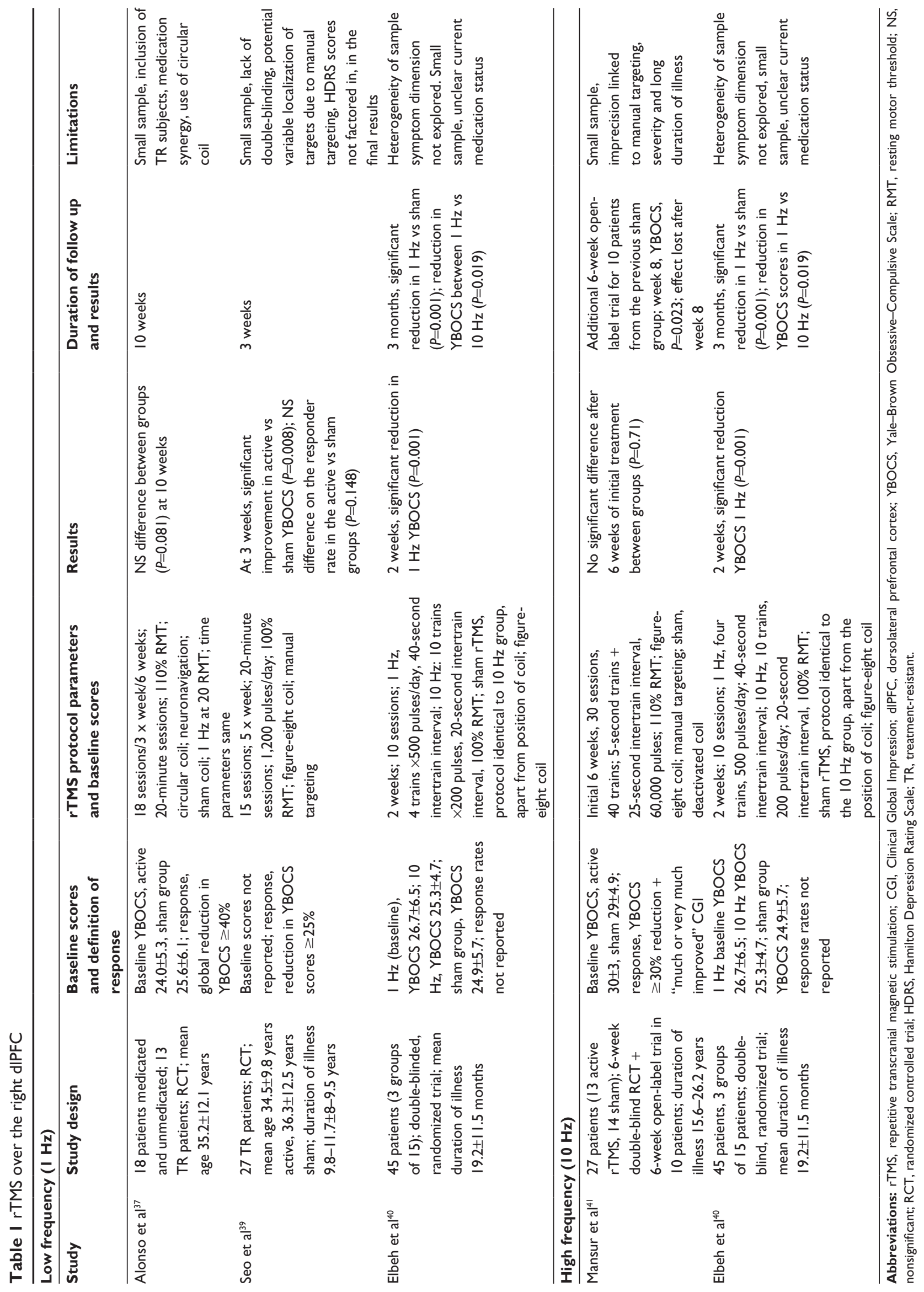


and sham (YBOCS 23.42 \pm 4.9 ) groups, which was taken into account in a final comparison between groups (Table 2).

High-frequency ( $\geq 5 \mathrm{~Hz}$ ) rTMS over the left dIPFC In 2007, Sachdev et al compared $10 \mathrm{~Hz}$ rTMS to sham rTMS over the left dlPFC in 18 TR OCD patients. ${ }^{43}$ Stimulation was applied over 10 sessions using a figure-eight coil, with an open extension to 20 sessions. The same parameters were used for the sham coil, positioned at $1 \mathrm{~m}$ from the scalp. The mean duration of illness for both groups was approximately 12 years, with mean ages $29.5 \pm 9.9$ years in the active and $35.8 \pm 8.2$ years in the sham group. There was no significant difference between groups on YBOCS scores at 2 or 4 weeks $(P=0.76, P=0.24)$ of treatment, even after correcting for Montgomery-Åsberg Depression Rating Scale (MADRS) scores, which improved after 4 weeks $(P=0.041)$. The study included a small sample of OCD patients with mild depressive disorder (Table 2).

\section{rTMS over bilateral dIPFC}

\section{Using HF $\alpha$-rTMS over bilateral dIPFC}

Ma et $\mathrm{al}^{44}$ used an innovative approach to stimulate the dIPFC using individualized rTMS frequency based on individually measured electroencephalography (EEG) $\alpha$-frequency. They compared $\alpha$-guided rTMS at $8-12 \mathrm{~Hz}$ frequencies with sham stimulation over 10 days in 46 medicated OCD patients: $\alpha$-rTMS sessions were 20 minutes long, delivered by a circular coil, using manual mapping of bilateral dlPFC. A sham coil was inactive, but with the same positioning as the active coil. Frequency of $\alpha$-rTMS was determined by average individual intrinsic $\alpha$-EEG frequency, resulting in delivery of 648-872 pulses per treatment. At week 2 , the $\alpha$-rTMS group showed significant reductions in YBOCS scores compared to the sham group $(P<0.01)$ and remained statistically significant at 1 week posttreatment for obsession ( $P=0.016)$, but not for compulsion subscores $(P=0.144)$. HARS scores showed significant reduction after 2 weeks of treatment $(P<0.01)$ and at 1 week follow-up $(P<0.01)$, but HDRS scores showed no significant changes between active and sham groups ( $P \geq 0.05)$. So far, this is the only published study to use individualized rTMS frequency and replication, and this would be interesting in a larger OCD group (Table 3).

\section{Studies using HF rTMS for bilateral dIPFC stimulation}

Haghighi et $\mathrm{al}^{45}$ and Jahangard et $\mathrm{al}^{46}$ analyzed $20 \mathrm{~Hz}$ rTMS applied sequentially over the left and right dlPFC in TR OCD patients. Both studies used a randomized single-blind crossover design to compare active rTMS to sham stimulation over 4 weeks. Both studies reported significant improvements in YBOCS scores in active versus sham groups. Limitations of both studies included single-blind design and lack

Table 2 LF and HF rTMS over the left dIPFC

\begin{tabular}{|c|c|c|c|c|c|c|}
\hline \multicolumn{7}{|c|}{ Low-frequency (I Hz) rTMS over the left dIPFC } \\
\hline Study & Study design & $\begin{array}{l}\text { Baseline scores } \\
\text { and definition of } \\
\text { response }\end{array}$ & $\begin{array}{l}\text { rTMS protocol } \\
\text { parameters and } \\
\text { baseline scores }\end{array}$ & Results & $\begin{array}{l}\text { Duration of } \\
\text { follow up } \\
\text { and results }\end{array}$ & Limitations \\
\hline Prasko et $\mathrm{al}^{42}$ & $\begin{array}{l}30 \text { TR OCD patients; } \\
\text { RCT; active vs sham; } \\
\text { age } 28.9-33.4 \text { years, } \\
\text { mean duration of } \\
\text { illness } ~ 15 \text { years }\end{array}$ & $\begin{array}{l}\text { YBOCS, active } \\
29.82 \pm 5.8 \text {, sham } \\
23.42 \pm 4.9 ; \\
\text { response } \\
\text { definition NA }\end{array}$ & $\begin{array}{l}2 \text { weeks, } 30 \text {-minute } \\
\text { sessions, I,800 pulses/ } \\
\text { session; I I0\% RMT; } \\
\text { sham stimulation, same } \\
\text { parameters at } 90^{\circ} \\
\text { over the left dIPFC; } \\
\text { figure-eight coil; manual } \\
\text { targeting }\end{array}$ & $\begin{array}{l}2 \text { weeks; NS } \\
\text { difference } \\
\text { between } \\
\text { groups }\end{array}$ & $\begin{array}{l}4 \text { weeks, } \\
\text { NS effects } \\
\text { on YBOCS, } \\
\text { CGI-S, HARS } \\
\text { between } \\
\text { groups }\end{array}$ & $\begin{array}{l}\text { Small sample, } \\
\text { baseline difference } \\
\text { on YBOCS scores } \\
\text { between active } \\
\text { and sham groups, } \\
\text { manual targeting of } \\
\text { left dIPFC, active } \\
\text { sham coil }\end{array}$ \\
\hline \multicolumn{7}{|c|}{ High-frequency $(>5 \mathrm{~Hz})$ rTMS over the left dIPFC } \\
\hline Sachdev et $\mathrm{al}^{43}$ & $\begin{array}{l}\text { I8 TR OCD patients; } \\
\text { 2-week RCT + 2-week } \\
\text { open-label study; mean } \\
\text { age, active } 29.5 \pm 9.9 \text { years, } \\
\text { sham } 35.8 \pm 8.2 \text { years; } \\
\text { duration of illness, active } \\
\text { I2.6 } \pm 7.5 \text { years, sham } \\
12.3 \pm 5.4 \text { years }\end{array}$ & $\begin{array}{l}\text { Baseline YBOCS, } \\
\text { active } 25.8 \pm 5.7 \text {, } \\
\text { sham } 23.9 \pm 9.9 ; \\
\text { clinically significant } \\
\text { improvement } \\
\text { definition, } \\
\text { reduction in } \\
\text { YBOCS } \geq 40 \%\end{array}$ & $\begin{array}{l}\text { I0 sessions } / 2 \text { weeks }-20 \\
\text { sessions } / 4 \text { weeks; each } \\
\text { session } 30 \text { trains } \times 5 \\
\text { seconds }+25 \text {-second } \\
\text { intertrain interval; I I0\% } \\
\text { RMT; manual targeting }\end{array}$ & $\begin{array}{l}\text { NS at } 2 \text { weeks } \\
(P=0.76)\end{array}$ & $\begin{array}{l}\text { NS at } 4 \text { weeks } \\
(P=0.24) ; \\
\text { clinically } \\
\text { significant } \\
\text { improvement } \\
\text { in } 6 \text { patients }\end{array}$ & $\begin{array}{l}\text { Small sample, TR } \\
\text { illness in chronic } \\
\text { OCD patients }\end{array}$ \\
\hline
\end{tabular}

Abbreviations: rTMS, repetitive transcranial magnetic stimulation; dIPFC, dorsolateral prefrontal cortex; TR, treatment-resistant; OCD, obsessive-compulsive disorder; RCT, randomized controlled trial; YBOCS, Yale-Brown Obsessive-Compulsive Scale; NA, not applicable; RMT, resting motor threshold; NS, nonsignificant; CGI-S, Clinical Global Impression - severity; HARS, Hamilton Anxiety Rating Scale. 


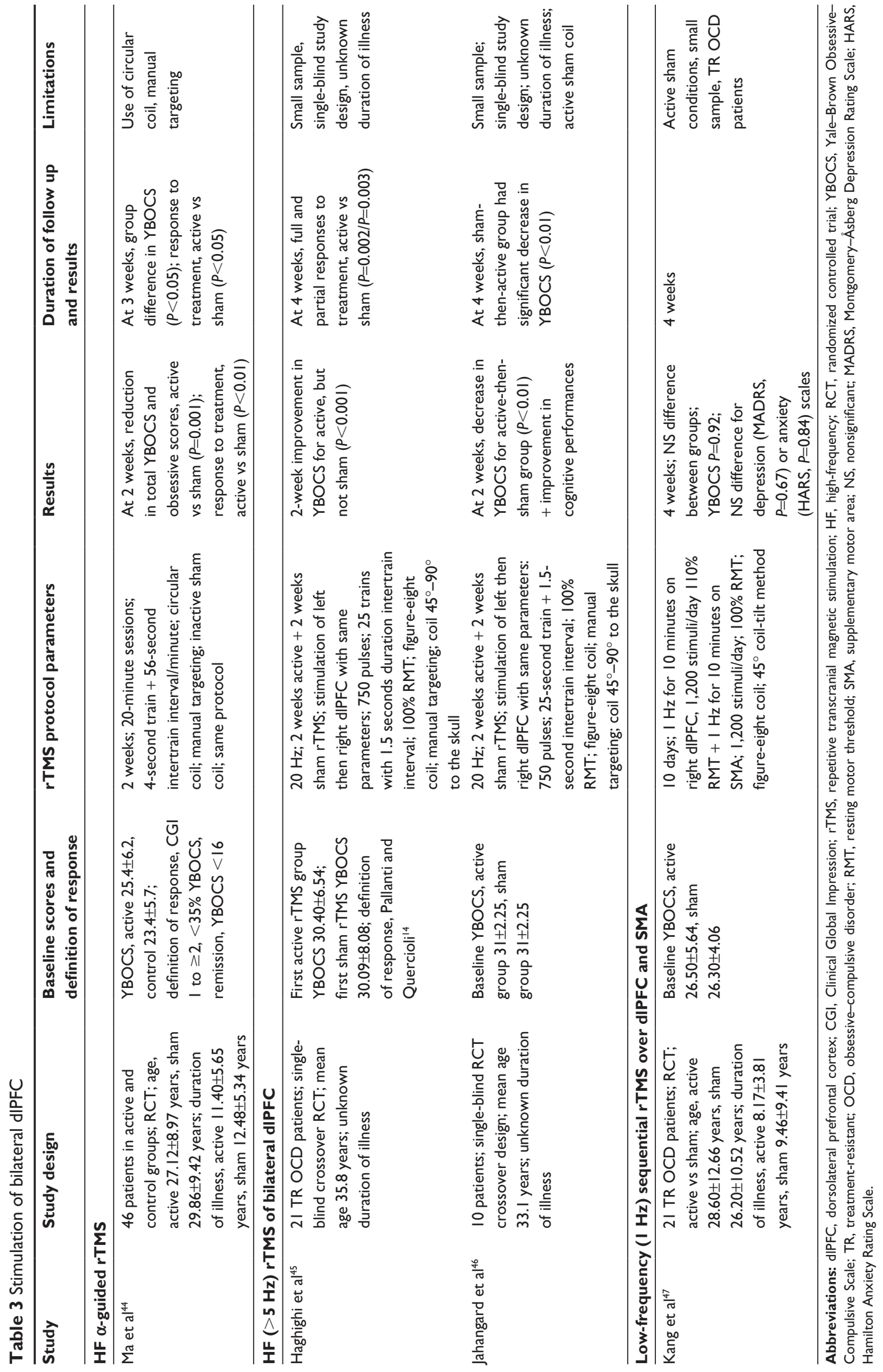


of monitoring for anxiety and depression symptoms, which can potentially moderate the intensity of OCD symptoms (Table 3).

Studies using LF sequential rTMS over the right dIPFC and SMA

In an RCT with 20 TR OCD patients, Kang et al ${ }^{47}$ assessed sham or $1 \mathrm{~Hz}$ stimulation over the right dlPFC at $110 \%$ resting motor threshold (RMT) for 10 minutes, followed by 10 minutes of SMA stimulation with $1 \mathrm{~Hz}$ at $100 \%$ RMT, over a period of 10 days. Sham stimulation was applied using a coil-tilt method, positioned at $45^{\circ}$ toward the scalp. Duration of OCD in both patient groups was $8.17 \pm 3.81-9.46 \pm 9.41$ years. There was a significant effect of time on YBOCS scores in each group $(P=0.009)$, but no significant difference between groups $(P=0.92)$. There was no difference between groups on depression (MADRS) or anxiety (HARS) scores. Limitations included small groups and potential effects of sham stimulation (Table 3).

In summary, stimulation of exclusively right or left dlPFC has not shown consistent data on the effectiveness of either HF or LF stimulation in OCD. Differences in methodology of reviewed studies including illness heterogeneity, stage of illness, and medication status, which have impacted the outcomes. More consistent data are accumulating on HF rTMS of the bilateral dIPFC, ${ }^{45,46}$ and an innovative $\alpha$-rTMS protocol over the bilateral dIPFC offers a promising way of individualizing HF stimulation of dlPFC and calls for replication. ${ }^{44}$

Interestingly, positive findings on LF stimulation over the right $\mathrm{dIPFC}^{39,40}$ were associated with a reduction in depressive and anxiety scores, an effect previously shown in LF rTMS stimulation over the right dIPFC in depression. Neurobiologically, there is insufficient understanding of deficits in the dorsal cognitive loop in OCD to guide the targeting of this circuitry in treatment. Identifying specific OCD symptom dimensions that respond to dIPFC stimulation and any comorbidities (eg, depression) that may be more likely to respond to stimulation over the dIPFC could inform future use of rTMS in OCD patients.

\section{rTMS over the SMA}

rTMS stimulation over the pre-SMA and SMA in OCD patients was first explored by Mantovani et al..$^{48}$ The pre-SMA (BA32/BA8) and SMA (BA 6) form a part of the sensorimotor CSTC circuit, together with the motor (M1) and premotor cortices. These cortical regions project topographically to the putamen, which in turn projects by direct (via globus pallidus interna) and indirect (via globus pallidus externa) pathways via basal ganglia to the thalamus and cortex, forming a sensorimotor CSTC loop. . $^{17,18,49}$

Research has delineated that rostral "associative" and caudal "sensorimotor" components of the sensorimotor cortex serve different functions in the process of planning and executing motor movements. ${ }^{50,51}$ The rostral, associative part of the sensorimotor cortex, including the pre-SMA, rostral cingulate motor area, and rostral premotor areas, are thought to be involved in sequence generation and motor learning, and have close connections with other PFC regions. ${ }^{50}$ Caudal components, including the SMA, motor cortex, caudal cingulate motor area, and premotor areas, are considered responsible for movement execution. ${ }^{18}$ The SMA is thought to be involved in internal generation of movement and construction of motor sequences with its direct projections to M1, spinal cord, and striatum..$^{50}$ The pre-SMA subsumes the resolution of competing motor plans ${ }^{52}$ by mechanisms of updating and implementing competing response plans. ${ }^{53}$ Functional alteration of the sensorimotor loop in OCD might be responsible for interfering with suppression of "internally triggered intrusive and repetitive movements and thoughts". ${ }^{20}$

There is a paucity of neuroimaging data on sensorimotor areas in OCD. Most of the evidence for abnormalities in the sensorimotor CTSC loop in OCD is underpinned by structural and functional neuroimaging studies focusing on different target areas or using different symptomprovocation/neurocognitive paradigms ${ }^{54-59}$ Findings of high motor excitability, structural deficits, and hyperactivity of the pre-SMA/SMA region in functional studies informed the use of inhibitory frequencies in rTMS stimulation of the SMA (Table 4).

Mantovani et a ${ }^{60}$ revisited the effects of $1 \mathrm{~Hz}$ rTMS over the SMA in 2010, following neuroimaging/spectroscopy findings of hyperactivity in premotor areas (including the SMA) and the dorsal ACC in OCD. ${ }^{61}$ In a 4-week doubleblind study followed by a 4-week open-label trial, 18 patients were initially randomly assigned to active rTMS or sham rTMS. In the open-label trial, nonresponders to sham rTMS and responders to active or sham rTMS were offered additional active rTMS for 4 weeks. Groups were followed every 2 weeks for 3 months with YBOCS and YBOCS self-reports as primary outcome measures. YBOCS results at baseline, week $2(P=0.294)$, and week $4(P=0.153)$ were not statistically significant between groups, but scores on YBOCS self-report scale $(P=0.031)$ and CGI-S for the active group $(P=0.044)$ improved after 4 weeks of treatment. The group who received 8 weeks of active rTMS had a statistically 


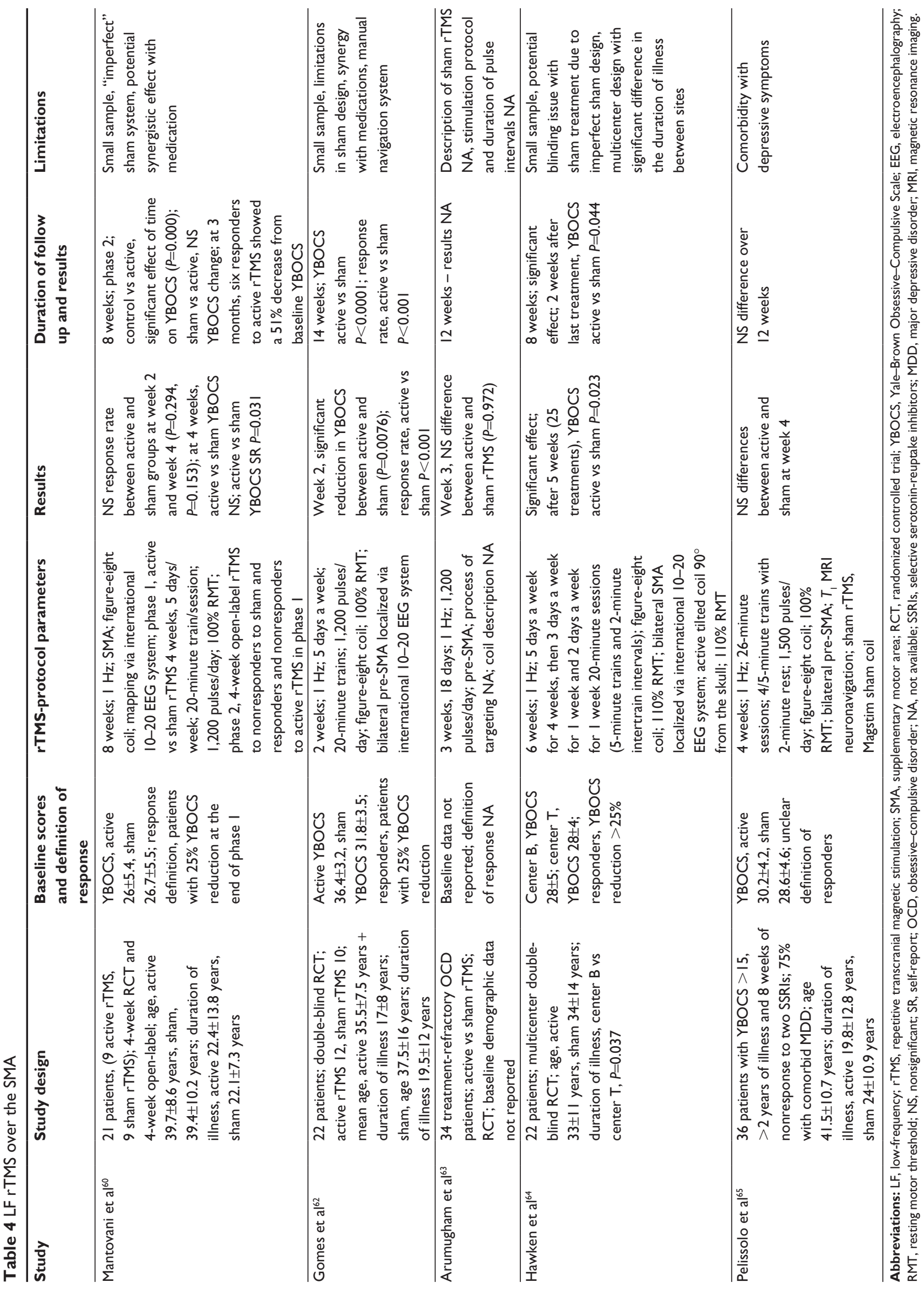


significant decrease in YBOCS $(P=0.000)$, while the sham rTMS group did not achieve statistically significant improvement in OCD symptoms when assigned to 4 weeks of active treatment, although they showed a significant improvement in general anxiety symptoms (HARS, $P=0.033$ ). Righthemisphere MT and right-left symmetry improved after active rTMS, but no significant change was observed in groups receiving sham rTMS. The authors suggested that it remained unclear whether baseline hyperactivation of motor and premotor areas was a primary alteration or a compensatory state reflecting neuronal deficit in another part of CTSC neurocircuitry.

In a 2-week, double-blind RCT, Gomes et $\mathrm{al}^{62}$ applied 1 $\mathrm{Hz}$ rTMS over the bilateral pre-SMA in 22 OCD patients, measuring YBOCS scores after active vs sham treatment. After 2 weeks of treatment, patients in the active group showed a significant reduction in YBOCS scores by $15.3 \pm 2.4$ points compared to $5.3 \pm 2.6$ points in the sham group $(P=0.0001)$. Reduction in YBOCS scores persisted for 14 weeks posttreatment in the active rTMS group. Initial improvement in anxiety scores on HARS, statistically significant for both groups, did not persist at 14 weeks, and there was no change in depression HDRS scores at the 2 nd or 14 th week for either group. Small samples, sham rTMS design, manual targeting of the pre-SMA region, and possible synergy with pharmacological treatment present methodological limitations of this study. In an RCT with 34 TR OCD patients, Arumugham et $\mathrm{al}^{63}$ applied $1 \mathrm{~Hz}$ rTMS or sham treatment over the bilateral pre-SMA for 3 weeks. There was no difference between groups on YBOCS scores $(P=0.972)$ after 3 weeks of treatment. Lack of baseline demographic data and baseline YBOCS and no description of the sham protocol allow limited interpretation of the findings.

In a 6-week, multicenter placebo RCT, Hawken et $\mathrm{al}^{64}$ examined $1 \mathrm{~Hz}$ rTMS in 22 TR OCD patients. The protocol consisted of daily rTMS in 20-minute sessions 5 days a week for the initial 4 weeks, reduced to 3 days a week in the fifth week, and twice a week in the sixth week of treatment. The bilateral SMA was stimulated after manual targeting via the international 10-20 EEG system. After 25 treatments, the group receiving active rTMS showed significant reduction in YBOCS scores $(P=0.023)$, and $80 \%$ of participants experienced a clinical response (YBOCS reduction $>25 \%$ ). Reduction in YBOCS scores in the active group persisted for 2 weeks posttreatment $(P=0.044)$. The active rTMS group also showed a significant reduction in depression scores measured by HDRS ( $P=0.021)$ without influencing YBOCS results. Limitations of the study included a small sample, significant difference in the duration of illness between groups $(P=0.037)$, lack of end-of-treatment follow-up for one center, and imperfect sham design. Pelissolo et al ${ }^{65}$ conducted an RCT of $1 \mathrm{~Hz}$ rTMS over the pre-SMA in 36 TR OCD patients over 4 weeks. At the end of week 4 , there was no difference between responders $(P=0.63)$ and completers $(P=0.47)$. It is worth noting that $75 \%$ of subjects had comorbid major depressive disorder (DSM-IV-TR), and there was no difference in depression scores measured by MADRS between groups after treatment. The authors proposed that having a severely TR sample with comorbid depression might explain the negative results (Table 4).

In summary, it appears that the majority of studies using the inhibitory $1 \mathrm{~Hz}$ rTMS over the bilateral pre-SMA/SMA have shown positive but short-term effects on reducing obsessive and compulsive symptoms. Generalizing study findings are restricted by small samples and a great deal of heterogeneity in severity of OCD symptoms, duration of illness, study designs, and methodology. Most importantly, stimulation protocols differed between studies in the duration of trains and intertrain intervals, the total number of pulses in a session, duration of treatment, and the delivery at different RMT thresholds. All these parameters influence the direction of stimulation and the duration of the effect. Although ideal stimulation parameters are so far unknown, studies using protocols consisting of 20-minute trains with 1,200 pulses/ day and at least 2 weeks' treatment seem to have resulted in positive outcomes on YBOCS scores.

\section{rTMS of the OFC}

Situated at a ventral bottom portion of the frontal lobes, the OFC forms a cortical part of the "ventral cognitive circuit" and is closely linked to the "affective CSTC circuit". ${ }^{19,66}$ Lesions to the OFC interfere with appropriate decisionmaking and response selection and impair adaptive response selection. ${ }^{17}$ OCD symptomatology is thought to arise as a result of excessive activity in parts of the ventral cognitive circuit, with the inability to resolve intrusive obsessions, and interrupt urges and anxiety that lead to repetitive compulsive behaviors. ${ }^{16,23}$ Table 5 summarizes the studies of rTMS in OCD targeting the OFC (Table 5).

Ruffini et $\mathrm{al}^{67}$ stimulated the OFC with active or sham rTMS in 23 TR OCD patients on medication, 5 days a week, for three consecutive weeks. Active stimulation was performed using a figure-of-eight-shaped $70 \mathrm{~mm}$ coil positioned over the left OFC (localized via international EEG system at Fp1). There was a significant reduction in YBOCS scores between the active and sham groups $(P<0.04)$, with significant 
Table 5 LF rTMS stimulation of OFC

\begin{tabular}{|c|c|c|c|c|c|c|}
\hline Study & Study design & $\begin{array}{l}\text { Baseline scores } \\
\text { and definition } \\
\text { of response }\end{array}$ & $\begin{array}{l}\text { rTMS protocol } \\
\text { parameters }\end{array}$ & Results & $\begin{array}{l}\text { Duration of } \\
\text { follow up } \\
\text { and results }\end{array}$ & Limitations \\
\hline Ruffini et $a^{67}$ & $\begin{array}{l}23 \text { TR OCD } \\
\text { patients; active } \\
\text { vs sham; baseline } \\
\text { YBOCS scores }>30 \\
\text { in both groups; age } \\
\text { NA; duration of } \\
\text { illness NA }\end{array}$ & $\begin{array}{l}\text { Baseline YBOCS } \\
\text { scores }>30 \text { in } \\
\text { both groups; } \\
\text { response } \\
\text { definition not } \\
\text { stated }\end{array}$ & $\begin{array}{l}\text { I5 days/I5 sessions; } \\
\text { I0-minute sessions; I Hz; } \\
\text { left OFC; figure-eight coil; } \\
\text { manual targeting; } 80 \% \text { RMT; } \\
\text { sham coil, inactive rTMS coil } \\
\text { placed perpendicularly to the } \\
\text { left OFC }\end{array}$ & $\begin{array}{l}\text { I } 5 \text { days; } \\
\text { active vs sham } \\
P<0.04\end{array}$ & $\begin{array}{l}3 \text { months; } \\
\text { significant } \\
\text { difference } \\
\text { until week } 10 \\
(P<0.06)\end{array}$ & \\
\hline Nauczyciel et $a^{68}$ & $\begin{array}{l}\text { I9 TR OCD } \\
\text { patients; RCT } \\
\text { crossover design; } \\
\text { on-off } 39 \text { (24-56); } \\
\text { off-on } 40 \text { (24-56) }\end{array}$ & $\begin{array}{l}\text { On-off YBOCS } \\
32 \text { (I5-36); off- } \\
\text { on } 32 \text { (16-36); } \\
\text { level of response } \\
\text { not measured }\end{array}$ & $\begin{array}{l}\text { I0 sessions/ } 2 \times \text { day in the } \\
\text { first week; I Hz; I } 20 \% \text { RMT; } \\
\text { I,200 pulses/session; right } \\
\text { OFC; D8- } 80 \text { butterfly double- } \\
\text { cone coil; sham coil placebo }\end{array}$ & $\begin{array}{l}\text { YBOCS active } \\
\text { vs sham } P=0.07\end{array}$ & $\begin{array}{l}35 \text { days; } \\
\text { active vs sham } \\
\text { YBOCS } P=0.94\end{array}$ & $\begin{array}{l}\text { Small sample, } \\
\text { design prone } \\
\text { to unblinding }\end{array}$ \\
\hline
\end{tabular}

Abbreviations: LF, low-frequency; rTMS, repetitive transcranial magnetic stimulation; OFC, orbitofrontal cortex; TR, treatment-resistant; OCD, obsessive-compulsive disorder; YBOCS, Yale-Brown Obsessive-Compulsive Scale; NA, not available; RMT, resting motor threshold; RCT, randomized controlled trial.

difference between active and sham stimulation measured at 10 weeks post-rTMS $(P<0.02)$. However, the effect was lost at a 10 -week follow-up $(P<0.06)$. There was no significant decrease in depression or anxiety scores between the groups. In terms of response, 15 of 16 patients receiving active rTMS had a $>35 \%$ reduction in YBOCS scores. The small sample, stimulation at $80 \%$ RMT, lack of precise localization of the target, and lack of supporting neuroimaging data ensuring OFC were indeed targeted and accessible to rTMS call for replication of the study. In a randomized double-blind crossover trial, Nauczyciel et $\mathrm{a}^{68}$ employed active vs sham rTMS targeted to the right OFC in 19 TR OCD patients with DB-80 coil. Ten patients underwent resting-state positron emission tomography scans: one after active stimulation sequence and one after sham stimulation. YBOCS scores decreased after 1 week in the active $(P<0.01)$ and sham groups $(P=0.02)$, with a trend toward a greater decrease in scores after active compared to sham stimulation $(P=0.07)$. Differences in YBOCS scores between groups dissipated at 35 days. There were no changes in MADRS scores over time. Positron emission tomography scan findings of the 10 patients after the active stimulation showed significant decreases in metabolism in the right frontal lobe (BA9), middle gyrus (BA10), orbital gyrus (BA47, BA11), left ACC (BA25), left frontal lobe (BA11), and left putamen. There were no brain regions with increased metabolism under active stimulation conditions. Decreased YBOCS scores correlated with a decrease in the metabolic activity of the right OFC (BA47).

\section{dTMS}

The development of dTMS in OCD emerged after the observed effectiveness of deep brain stimulation on subcortical structures in OCD patients, in addition to exploring noninvasive methods of stimulating deep brain structures. ${ }^{69}$ Double-cone and $\mathrm{H}$ coils have been developed to reach and stimulate deeper subcortical structures. ${ }^{70}$ dTMS targets of interest in OCD have been the mPFC and ACC, consistently implicated in OCD CSTC neurocircuitry by neuroimaging findings and observations of improvement in OCD symptoms after anterior cingulotomy or anterior capsulotomy. ${ }^{71}$ So far there have been two published RCTs on the use of dTMS in OCD. Carmi et al observed the effects on OCD symptoms after stimulating the $\mathrm{mPFC}$ and $\mathrm{ACC},{ }^{72}$ while Nauczyciel et al examined the effects of targeting $\mathrm{OFC}^{68}$ (Table 6).

In a double-blind controlled study, Carmi et al ${ }^{72}$ used dTMS H7-coil or sham treatment over 5 weeks in 41 OCD patients on SRIs. The majority of patients had OCD for $>10$ years and were in the 25- to 40-year age group. Patients were randomized to $20 \mathrm{~Hz}, 1 \mathrm{~Hz}$, or sham treatment, and target areas were the $\mathrm{mPFC}$ and ACC. The group receiving $20 \mathrm{~Hz}$ dTMS improved significantly in YBOCS scores compared to the $1 \mathrm{~Hz}$ and placebo groups (28\% vs 6\% reduction, $\left.t_{93}=-2.29 ; P=0.0243\right)$. YBOCS scores recorded over the following 3 months remained stable. The $\mathrm{H} 7$ coil used by Carmi et al is designed to reach the MPFC and ACC. A D8-80 butterfly double-cone coil had been used previously with a positive effect over the right OFC.$^{68}$ Pairing dTMS stimulation with functional neuroimaging tools would improve the reliability of reaching intended targets and offer the possibility of correlating neuroimaging findings with any potential clinical effects. Concurrent stimulation of superficial cortical areas with dTMS devices might contribute to treatment effects (Table 6). 
Table $6 \mathrm{HF}$ and LF stimulation via dTMS

\begin{tabular}{|c|c|c|c|c|c|c|}
\hline Study & Study design & $\begin{array}{l}\text { Baseline scores } \\
\text { and definition } \\
\text { of response }\end{array}$ & $\begin{array}{l}\text { rTMS protocol } \\
\text { parameters and } \\
\text { baseline scores }\end{array}$ & Results & $\begin{array}{l}\text { Duration of } \\
\text { follow up } \\
\text { and results }\end{array}$ & Limitations \\
\hline Carmi et $\mathrm{al}^{72}$ & $\begin{array}{l}\text { Double-blind, controlled } \\
\text { trial; } 4 \text { I OCD patients; } \\
\text { sham } 20 \mathrm{~Hz} \text {, active I Hz; + } \\
\text { simultaneous with } \\
\text { symptom provocation; age } \\
28 \pm 3.1 \text { years, } 35 \pm 3.5 \text { years, } \\
36 \pm 2.1 \text { years }\end{array}$ & $\begin{array}{l}\text { Baseline YBOCS } \\
25-28\end{array}$ & $\begin{array}{l}5 \text { weeks; I Hz/20 Hz; } \\
\text { targeting mPFC, including } \\
\text { ACC; LF, I Hz, I I0\% RMT, } \\
900 \text { pulses; HF, } 20 \mathrm{~Hz} \text {, } \\
\text { I00\% RMT, } 50 \text { 2-second } \\
\text { trains (2,000 pulses); H7 } \\
\text { coil; sham coil NA }\end{array}$ & $\begin{array}{l}\text { At } 5 \text { weeks, } \\
20 \mathrm{~Hz} \text { dTMS } \\
\text { vs I } \mathrm{Hz} \text { dTMS } \\
P=0.0243\end{array}$ & $\begin{array}{l}\text { At } 3 \text { months, } \\
\text { stability in } \\
\text { improvements } \\
\text { measured by } \\
\text { YBOCS }\end{array}$ & $\begin{array}{l}\text { Accessibility } \\
\text { of specific coil } \\
\text { for general use; } \\
\text { particular stimulation } \\
\text { protocol linked } \\
\text { with symptom } \\
\text { provocation }\end{array}$ \\
\hline
\end{tabular}

Abbreviations: HF, high-frequency; LF, low-frequency; dTMS, deep transcranial magnetic stimulation; OCD, obsessive-compulsive disorder; YBOCS, Yale-Brown Obsessive-Compulsive Scale; rTMS, repetitive TMS; mPFC, medial prefrontal cortex; ACC, anterior cingulate cortex; RMT, resting motor threshold; NA, not available.

\section{Discussion}

Electrical activity in the brain has been a source of interest for over two centuries, with electrophysiology emerging as a "pre-eminent method for the localization of brain functions" ${ }^{73}$ In recent years, the field of affective neuroscience has become richer, with the availability of sophisticated neuroimaging and new neurostimulation techniques that are able to detect, monitor, and correlate neural changes with affective, cognitive, and behavioral processes. Recognition that neural networks are interconnected and communicate at different levels has facilitated a better understanding of the neurobiological concepts related to psychiatric disorders. However, consistent treatment outcomes of complex psychiatric conditions, such as OCD, have been elusive. rTMS offers a treatment approach with a mechanism of action that can directly impact obsessive and compulsive symptom circuitry.

Stimulation of different brain areas, such as the dlPFC, SMA, OFC, and ACC, has so far elicited encouraging but mixed results, making it a potential add-on treatment strategy, though in need of refinement. A small number of studies suggest that HF stimulation of the right dlPFC, ${ }^{41,74}$ sequential HF stimulation of the bilateral dlPFC, ${ }^{45,46}$ and novel $\alpha$-guided rTMS using HF ranges ${ }^{44}$ may result in short-term improvement in OCD symptoms. This is in line with a recent meta-analysis ${ }^{30}$ reporting on the effectiveness of HF stimulation over the bilateral dlPFC - right dlPFC and left dlPFC - in improving OCD symptoms; however, previous systematic reviews and meta-analyses concluded that rTMS over the dIPFC was not more effective than sham stimulation. ${ }^{27,28,75-78}$

Outcomes have been more consistent for rTMS over the bilateral SMA, with significant beneficial effects noted as early as 4 weeks and lasting for up to 14 weeks. ${ }^{60,62}$ Interestingly, methodological heterogeneity comprising different inhibitory stimulation protocols, different OCD subtypes, and concomitant medication did not affect the findings in any profound way. One possible explanation could be that inhibitory rTMS over the SMA has a larger effect due to "stronger" connectivity with striatal regions compared to the dlPFC in OCD patients. Therefore, inhibition of the SMA might lead to short-term "normalization" of the SMA CTSC and interconnected regions, and subsequently help patients deal better with intrusive thoughts, impulses, and compulsions. $^{78}$

Two studies using LF inhibitory stimulation over the OFC yielded temporary but statistically significant positive results for OCD symptoms compared to sham stimulation in TR OCD patients. ${ }^{67,68}$ This is consistent with the findings of OFC hyperactivity in OCD. ${ }^{79}$ Previous studies have highlighted the promising acute therapeutic response of rTMS applied to OFC. ${ }^{28,30,76}$ In terms of dTMS, studies targeting the ACC and $\mathrm{mPFC}$ have shown positive effects on OCD symptoms using double-cone and $\mathrm{H} 7$ coil under different stimulation parameters and protocols. ${ }^{68,72}$ Stimulation via dTMS devices reaches varying brain surface areas and depths. ${ }^{70}$ Positive effects of dTMS could be conceptualized as a sum of all stimulated cortical and subcortical regions at the time of stimulation. Therefore, dTMS could likely surpass the rTMS limitations of cortical superficial stimulation by the extent of effect on subcortical circuits. However, the number of trials using dTMS is limited, and more research is required.

The interpretation and generalizability of findings from rTMS research in OCD is challenged by a number of design and methodological differences across studies. For example, there is a wide between-study variability in such patient characteristics as age of OCD onset, severity, OCD subtype, and of illness (months to years), treatment status, and stimulation protocols. Age of onset and duration of illness might impact the degree of response to rTMS, as both these factors have been associated with comorbidities and treatment resistance. ${ }^{80}$ Severe versus mild-moderate illness might 
additionally influence the extent of therapeutic rTMS effects, as shown in depression, where the severity of depression has been shown to predict the remission rates with rTMS, such that people with mild and moderate symptoms appeared to respond better to the treatment. ${ }^{81}$

A further challenge for the field is identifying which brain areas are responsible for mediating different OCD symptom dimensions. Such an understanding could inform which targets are most likely to be responsive to rTMS at an individual patient level. Further consideration also needs to be given to precise stimulation parameters. For example, Zhou et $\mathrm{a}^{30}$ showed that stimulation at $100 \%$ RMT was more effective than stimulation at other intensities across a number of studies. Variability in sham protocols between studies has been shown to impact treatment effect sizes, depending on the use of active or inactive coil. ${ }^{30}$ The underlying mechanisms are unclear, especially considering a generally low placebo effect in severe OCD. ${ }^{82}$

Meta-analyses ${ }^{27-31}$ of TMS in OCD have attempted to aggregate data from a number of different studies. Though relevant in generating information on promising targets and stimulation parameters, combined effect results have limitations in enhancing our understanding of clinical predictors (age, duration of OCD, baseline medication) and stimulationrelevant factors (eg, target, frequency range, and stimulation frequency) that are potentially associated with positive treatment outcomes. In addition, it is not yet clear whether there are significant differences in efficacy between targets. The modest number of studies and clinical heterogeneity among studies calls for careful interpretation. Apart from the effect of rTMS on the SMA (use of only LF stimulation on SMA), which yields a moderate effect size, results from other metaanalyses combining HF and LF parameters and different stimulation duration, as well as different sham conditions, are not specific enough to guide clinical practice.

The majority of rTMS studies in OCD have been conducted in TR OCD patients as an augmentation strategy to standard pharmacological treatment. There is a lack of studies comparing other augmentation strategies with rTMS as an add-on treatment in this subgroup of patients. ${ }^{83}$ There is a need to address the design and methodological heterogeneity of rTMS studies, including the difference in stimulation protocols, the underlying variability in pharmacological treatment, and the heterogeneity of OCD subtypes, which might have obscured the true therapeutic effect of rTMS. Addressing these issues will help clarify to what extent rTMS is clinically comparable with other augmentation strategies and thus valuable in the OCD treatment armamentarium.
Larger studies will also provide more statistical power to detect meaningful effects of treatment.

In conclusion, as a new neurostimulation technique, rTMS shows promise as part of a toolbox of current psychiatric treatment options for OCD. Its noninvasiveness with good tolerability and side-effect profile make it an appealing treatment consideration. Underlying neurobiological mechanisms related to TMS are still under evaluation, but appear to offer a novel "third" way of addressing symptoms via localized electrical stimulation compared to pharmacotherapy and psychotherapy approaches. Recent studies have considered rTMS as an adjunct treatment in TR patients with long duration of illness. In future, apart from managing design and methodology issues encountered in previous studies, it will be of interest to address rTMS as an augmentation strategy earlier in the course of treatment and consider parallel stimulation of a few cortical regions. Also, rTMS could be evaluated as an option in medication-naïve patients who cannot tolerate pharmacological treatment and have limited benefits from psychotherapy approaches. Advances in targeted brain stimulation via development of dTMS H coils paired with functional neuroimaging are offering a step further in understanding and management of OCD. However, it is yet to be determined how we can best optimize the approach via rTMS or dTMS to achieve clinically relevant results.

\section{Disclosure}

The authors report no conflicts of interest in this work.

\section{References}

1. American Psychiatric Association. Diagnostic and Statistical Manual of Mental Disorders. 5th ed. Washington, DC: APA; 2013.

2. World Health Organization. The ICD-10 Classification of Mental and Behavioural Disorders: Clinical Descriptions and Diagnostic Guidelines. Geneva: WHO; 1992.

3. Subramaniam M, Soh P, Vaingankar JA, Picco L, Chong SA. Quality of life in obsessive-compulsive disorder: impact of the disorder and of treatment. CNS Drugs. 2013;27(5):367-383.

4. Kessler RC, Berglund P, Demler O, Jin R, Merikangas KR, Walters EE. Lifetime prevalence and age-of-onset distributions of DSM-IV disorders in the National Comorbidity Survey Replication. Arch Gen Psychiatry. 2005;62(6):593-602

5. Ruscio AM, Stein DJ, Chiu WT, Kessler RC. The epidemiology of obsessive-compulsive disorder in the National Comorbidity Survey Replication. Mol Psychiatry. 2010;15(1):53-63.

6. Castle DJ, Deale A, Marks IM. Gender differences in obsessive compulsive disorder. Aust N Z J Psychiatry. 1995;29(1):114-117.

7. Jenike MA. Clinical practice: obsessive-compulsive disorder. $N$ Engl J Med. 2004;350(3):259-265.

8. Goodman WK, McDougle CJ, Barr LC, Aronson SC, Price LH. Biological approaches to treatment-resistant obsessive compulsive disorder. J Clin Psychiatry. 1993;54(Suppl):16-26.

9. Schruers K, Koning K, Luermans J, Haack MJ, Griez E. Obsessivecompulsive disorder: a critical review of therapeutic perspectives. Acta Psychiatr Scand. 2005;111(4):261-271. 
10. Koran LM, Hanna GL, Hollander E, Nestadt G, Simpson HB. Practice guideline for the treatment of patients with obsessive-compulsive disorder. Am J Psychiatry. 2007;164(7 Suppl):5-53.

11. Bandelow B, Zohar J, Hollander E, et al. World Federation of Societies of Biological Psychiatry (WFSBP) guidelines for the pharmacological treatment of anxiety, obsessive-compulsive and post-traumatic stress disorders: first revision. World J Biol Psychiatry. 2008;9(4):248-312.

12. Baldwin DS, Anderson IM, Nutt DJ, et al. Evidence-based pharmacological treatment of anxiety disorders, post-traumatic stress disorder and obsessive-compulsive disorder: a revision of the 2005 guidelines from the British Association for Psychopharmacology. J Psychopharmacol. 2014;28(5):403-439.

13. Pallanti S, Hollander E, Bienstock C, et al. Treatment non-response in OCD: methodological issues and operational definitions. Int J Neuropsychopharmacol. 2002;5(2):181-191.

14. Pallanti S, Quercioli L. Treatment-refractory obsessive-compulsive disorder: methodological issues, operational definitions and therapeutic lines. Prog Neuropsychopharmacol Biol Psychiatry. 2006;30(3): 400-412.

15. Castle D, Bosanac P, Rossell S. Treating OCD: what to do when firstline therapies fail. Australas Psychiatry. 2015;23(4):350-353.

16. Milad MR, Rauch SL. Obsessive-compulsive disorder: beyond segregated cortico-striatal pathways. Trends Cogn Sci. 2012;16(1):43-51.

17. Alexander GE, DeLong MR, Strick PL. Parallel organization of functionally segregated circuits linking basal ganglia and cortex. Annu Rev Neurosci. 1986;9:357-381.

18. Haber SN. The primate basal ganglia: parallel and integrative networks. J Chem Neuroanat. 2003;26(4):317-330.

19. van den Heuvel OA, van Wingen G, Soriano-Mas C, et al. Brain circuitry of compulsivity. Eur Neuropsychopharmacol. 2016;26(5):810-827.

20. Russo M, Naro A, Mastroeni C, et al. Obsessive-compulsive disorder: a "sensory-motor" problem? Int J Psychophysiol. 2014;92(2):74-78.

21. Sheth SA, Mian MK, Patel SR, et al. Human dorsal anterior cingulate cortex neurons mediate ongoing behavioural adaptation. Nature. 2012;488(7410):218-221.

22. Vahabzadeh A, McDougle CJ. Obsessive-compulsive disorder. In: McManus LM, Mitchell RN, editors. Pathobiology of Human Disease. San Diego, CA: Academic Press; 2014:2512-2521.

23. Maia TV, Cooney RE, Peterson BS. The neural bases of obsessivecompulsive disorder in children and adults. Dev Psychopathol. 2008; 20(4):1251-1283.

24. Comte M, Schön D, Coull JT, et al. Dissociating bottom-up and top-down mechanisms in the cortico-limbic system during emotion processing. Cereb Cortex. 2016;26(1):144-155.

25. Chen R, Classen J, Gerloff C, et al. Depression of motor cortex excitability by low-frequency transcranial magnetic stimulation. Neurology. 1997;48(5):1398-1403.

26. Houdayer E, Degardin A, Cassim F, Bocquillon P, Derambure P, Devanne $H$. The effects of low- and high-frequency repetitive TMS on the input/output properties of the human corticospinal pathway. Exp Brain Res. 2008;187(2):207-217.

27. Slotema CW, Blom JD, Hoek HW, Sommer IE. Should we expand the toolbox of psychiatric treatment methods to include repetitive transcranial magnetic stimulation (rTMS)? A meta-analysis of the efficacy of rTMS in psychiatric disorders. J Clin Psychiatry. 2010;71(7): 873-884.

28. Berlim MT, Neufeld NH, van den Eynde F. Repetitive transcranial magnetic stimulation (rTMS) for obsessive-compulsive disorder (OCD): an exploratory meta-analysis of randomized and sham-controlled trials. J Psychiatr Res. 2013;47(8):999-1006.

29. Trevizol AP, Shiozawa P, Cook IA, et al. Transcranial magnetic stimulation for obsessive-compulsive disorder: an updated systematic review and meta-analysis. $J E C T$. 2016;32(4):262-266.

30. Zhou DD, Wang W, Wang GM, Li DQ, Kuang L. An updated metaanalysis: short-term therapeutic effects of repeated transcranial magnetic stimulation in treating obsessive-compulsive disorder. J Affect Disord. 2017;215:187-196.
31. Ma ZR, Shi LJ. Repetitive transcranial magnetic stimulation (rTMS) augmentation of selective serotonin reuptake inhibitors (SSRIs) for SSRIresistant obsessive-compulsive disorder (OCD): a meta-analysis of randomized controlled trials. Int J Clin Exp Med. 2014;7(12):4897-4905.

32. Alvarez JA, Emory E. Executive function and the frontal lobes: a metaanalytic review. Neuropsychol Rev. 2006;16(1):17-42.

33. MacDonald AW 3rd, Cohen JD, Stenger VA, Carter CS. Dissociating the role of the dorsolateral prefrontal and anterior cingulate cortex in cognitive control. Science. 2000;288(5472):1835-1838.

34. Robertson EM, Tormos JM, Maeda F, Pascual-Leone A. The role of the dorsolateral prefrontal cortex during sequence learning is specific for spatial information. Cereb Cortex. 2001;11(7):628-635.

35. Mataix-Cols D, van den Heuvel OA. Common and distinct neural correlates of obsessive-compulsive and related disorders. Psychiatr Clin North Am. 2006;29(2):391-410, viii.

36. Greenberg BD, George MS, Martin JD, et al. Effect of prefrontal repetitive transcranial magnetic stimulation in obsessive-compulsive disorder: a preliminary study. Am J Psychiatry. 1997;154(6):867-869.

37. Alonso P, Pujol J, Cardoner N, et al. Right prefrontal repetitive transcranial magnetic stimulation in obsessive-compulsive disorder: a double-blind, placebo-controlled study. Am J Psychiatry. 2001;158(7): $1143-1145$.

38. George MS, Belmaker RH. Transcranial Magnetic Stimulation in Clinical Psychiatry. Washington, DC: American Psychiatric Publishing; 2007.

39. Seo HJ, Jung YE, Lim HK, Um YH, Lee CU, Chae JH. Adjunctive low-frequency repetitive transcranial magnetic stimulation over the right dorsolateral prefrontal cortex in patients with treatment-resistant obsessive-compulsive disorder: a randomized controlled trial. Clin Psychopharmacol Neurosci. 2016;14(2):153-160.

40. Elbeh KA, Elserogy YM, Khalifa HE, Ahmed MA, Hafez MH, Khedr EM. Repetitive transcranial magnetic stimulation in the treatment of obsessive-compulsive disorders: double blind randomized clinical trial. Psychiatry Res. 2016;238:264-269.

41. Mansur CG, Myczkowki ML, Cabral SB, et al. Placebo effect after prefrontal magnetic stimulation in the treatment of resistant obsessivecompulsive disorder: a randomized controlled trial. Int J Neuropsychopharmacol. 2011;14(10):1389-1397.

42. Prasko J, Pasková B, Záleský R, et al. The effect of repetitive transcranial magnetic stimulation (rTMS) on symptoms in obsessive compulsive disorder: a randomized, double blind, sham controlled study. Neuro Endocrinol Lett. 2006;27(3):327-332.

43. Sachdev PS, Loo CK, Mitchell PB, McFarquhar TF, Malhi GS. Repetitive transcranial magnetic stimulation for the treatment of obsessive compulsive disorder: a double-blind controlled investigation. Psychol Med. 2007;37(11):1645-1649.

44. Ma X, Huang Y, Liao L, Jin Y. A randomized double-blinded shamcontrolled trial of $\alpha$ electroencephalogram-guided transcranial magnetic stimulation for obsessive-compulsive disorder. Chin Med J. 2014; 127(4):601-606.

45. Haghighi M, Shayganfard M, Jahangard L, et al. Repetitive transcranial magnetic stimulation (rTMS) improves symptoms and reduces clinical illness in patients suffering from OCD: results from a single-blind, randomized clinical trial with sham cross-over condition. J Psychiatr Res. 2015;68:238-244.

46. Jahangard L, Haghighi M, Shyayganfard M, et al. Repetitive transcranial magnetic stimulation improved symptoms of obsessive-compulsive disorder, but also cognitive performance: results from a randomized clinical trial with a cross-over design and sham condition. Neuropsychobiology. 2016;73(4):224-232.

47. Kang JI, Kim CH, Namkoong K, Lee CI, Kim SJ. A randomized controlled study of sequentially applied repetitive transcranial magnetic stimulation in obsessive-compulsive disorder. J Clin Psychiatry. 2009; 70(12):1645-1651.

48. Mantovani A, Lisanby SH, Pieraccini F, Ulivelli M, Castrogiovanni P, Rossi S. Repetitive transcranial magnetic stimulation (rTMS) in the treatment of obsessive-compulsive disorder (OCD) and Tourette's syndrome (TS). Int J Neuropsychopharmacol. 2005;9(1):95-100. 
49. Middleton FA. A revised neuroanatomy of frontal-subcortical circuits. In: Lichter DG, editor. Frontal-Subcortical Circuits in Psychiatric and Neurological Disorders. New York: Guildford Press; 2001:44-58.

50. Akkal D, Dum RP, Strick PL. Supplementary motor area and presupplementary motor area: targets of basal ganglia and cerebellar output. J Neurosci. 2007;27(40):10659-10673.

51. August JM, Rothenberger A, Baudewig J, Roessner V, Dechent P. May functional imaging be helpful for behavioral assessment in children? Regions of motor and associative cortico-subcortical circuits can be differentiated by laterality and rostrality. Front Hum Neurosci. 2015;9:314.

52. Nachev P, Wydell H, O’Neill K, Husain M, Kennard C. The role of the pre-supplementary motor area in the control of action. Neuroimage. 2007;36(Suppl 2):T155-T163.

53. Roberts RE, Husain M. A dissociation between stopping and switching actions following a lesion of the pre-supplementary motor area. Cortex. 2015;63:184-195

54. van den Heuvel OA, Remijnse PL, Mataix-Cols D, et al. The major symptom dimensions of obsessive-compulsive disorder are mediated by partially distinct neural systems. Brain. 2009;132(Pt 4):853-868.

55. Radua J, Mataix-Cols D. Voxel-wise meta-analysis of grey matter changes in obsessive-compulsive disorder. Br JPsychiatry. 2009;195(5): 393-402.

56. Gilbert AR, Akkal D, Almeida JR, et al. Neural correlates of symptom dimensions in pediatric obsessive-compulsive disorder: a functional magnetic resonance imaging study. J Am Acad Child Adolesc Psychiatry. 2009;48(9):936-944.

57. de Wit SJ, Alonso P, Schweren L, et al. Multicenter voxel-based morphometry mega-analysis of structural brain scans in obsessivecompulsive disorder. Am J Psychiatry. 2014;171(3):340-349.

58. de Wit SJ, de Vries FE, van der Werf YD, et al. Presupplementary motor area hyperactivity during response inhibition: a candidate endophenotype of obsessive-compulsive disorder. Am J Psychiatry. 2012;169(10): 1100-1108.

59. Eng GK, Sim K, Chen SH. Meta-analytic investigations of structural grey matter, executive domain-related functional activations, and white matter diffusivity in obsessive compulsive disorder: an integrative review. Neurosci Biobehav Rev. 2015;52:233-257.

60. Mantovani A, Simpson HB, Fallon BA, Rossi S, Lisanby SH. Randomized sham-controlled trial of repetitive transcranial magnetic stimulation in treatment-resistant obsessive-compulsive disorder. Int J Neuropsychopharmacol. 2010;13(2):217-227.

61. Yücel M, Harrison BJ, Wood SJ, et al. Functional and biochemical alterations of the medial frontal cortex in obsessive-compulsive disorder. Arch Gen Psychiatry. 2007;64(8):946-955.

62. Gomes PV, Brasil-Neto JP, Allam N, Rodrigues de Souza E. A randomized, double-blind trial of repetitive transcranial magnetic stimulation in obsessive-compulsive disorder with three-month follow-up. J Neuropsychiatry Clin Neurosci. 2012;24(4):437-443.

63. Arumugham SS, Subhasini VS, Madhuri HN, et al. Augmentation effect of low frequency-repetitive transcranial magnetic stimulation over pre-supplementary motor area in obsessive compulsive disorder: a randomized controlled trial. Brain Stimul. 2015;8(2):391.

64. Hawken ER, Dilkov D, Kaludiev E, Simek S, Zhang F, Milev R. Transcranial magnetic stimulation of the supplementary motor area in the treatment of obsessive-compulsive disorder: a multi-site study. Int $J$ Mol Sci. 2016;17(3):420.

65. Pelissolo A, Harika-Germaneau G, Rachid F, et al. Repetitive transcranial magnetic stimulation to supplementary motor area in refractory obsessive-compulsive disorder treatment: a sham-controlled trial. Int J Neuropsychopharmacol. 2016;19(8):pyw025.
66. Pauls DL, Abramovitch A, Rauch SL, Geller DA. Obsessive-compulsive disorder: an integrative genetic and neurobiological perspective. Nat Rev Neurosci. 2014;15(6):410-424.

67. Ruffini C, Locatelli M, Lucca A, Benedetti F, Insacco C, Smeraldi E. Augmentation effect of repetitive transcranial magnetic stimulation over the orbitofrontal cortex in drug-resistant obsessive-compulsive disorder patients: a controlled investigation. Prim Care Companion J Clin Psychiatry. 2009;11(5):226-230.

68. Nauczyciel C, le Jeune F, Naudet F, et al. Repetitive transcranial magnetic stimulation over the orbitofrontal cortex for obsessivecompulsive disorder: a double-blind, crossover study. Transl Psychiatry. 2014;4:e436.

69. Alonso P, Cuadras D, Gabriëls L, et al. Deep brain stimulation for obsessive-compulsive disorder: a meta-analysis of treatment outcome and predictors of response. PLoS One. 2015;10(7):e0133591.

70. Tendler A, Ygael NB, Roth Y, Zangen A. Deep transcranial magnetic stimulation (dTMS): beyond depression. Expert Rev Med Devices. 2016;13(10):987-1000.

71. Greenberg BD, Murphy DL, Rasmussen SA. Neuroanatomically based approaches to obsessive-compulsive disorder: neurosurgery and transcranial magnetic stimulation. Psychiatr Clin North Am. 2000;23(3): 671-686.

72. Carmi L, al Yagon U, Dar R, Zohar J, Zangen A. Deep transcranial magnetic stimulation (TMS) in obsessive compulsive disorder (OCD) patients. Eur Psychiatry. 2015;30(Suppl 1):794.

73. Brazier MA. The history of the electrical activity of the brain as a method for localizing sensory function. Med Hist. 1963;7:199-211.

74. Sachdev PS, McBride R, Loo CK, Mitchell PB, Malhi GS, Croker VM. Right versus left prefrontal transcranial magnetic stimulation for obsessive-compulsive disorder: a preliminary investigation. J Clin Psychiatry. 2001;62(12):981-984.

75. Martin JL, Barbanoj MJ, Pérez V, Sacristán M. Transcranial magnetic stimulation for the treatment of obsessive-compulsive disorder. Cochrane Database Syst Rev. 2003;(3):CD003387.

76. Blom RM, Figee M, Vulink N, Denys D. Update on repetitive transcranial magnetic stimulation in obsessive-compulsive disorder: different targets. Curr Psychiatry Rep. 2011;13(4):289-294.

77. Jaafari N, Rachid F, Rotge JY, et al. Safety and efficacy of repetitive transcranial magnetic stimulation in the treatment of obsessive-compulsive disorder: a review. World J Biol Psychiatry. 2012;13(3):164-177.

78. Saba G, Moukheiber A, Pelissolo A. Transcranial cortical stimulation in the treatment of obsessive-compulsive disorders: efficacy studies. Curr Psychiatry Rep. 2015;17(5):36.

79. Rotge JY, Guehl D, Dilharreguy B, et al. Provocation of obsessivecompulsive symptoms: a quantitative voxel-based meta-analysis of functional neuroimaging studies. J Psychiatry Neurosci. 2008;33(5): 405-412.

80. Kalra SK, Swedo SE. Children with obsessive-compulsive disorder: are they just "little adults"? J Clin Invest. 2009;119(4):737-746.

81. Grammer GG, Kuhle AR, Clark CC, Dretsch MN, Williams KA, Cole JT. Severity of depression predicts remission rates using transcranial magnetic stimulation. Front Psychiatry. 2015;6:114.

82. Sugarman MA, Kirsch I, Huppert JD. Obsessive-compulsive disorder has a reduced placebo (and antidepressant) response compared to other anxiety disorders: a meta-analysis. J Affect Disord. 2017;218: 217-226.

83. Pallanti S, Marras A, Salerno L, Makris N, Hollander E. Better than treated as usual: transcranial magnetic stimulation augmentation in selective serotonin reuptake inhibitor-refractory obsessive-compulsive disorder, mini-review and pilot open-label trial. J Psychopharmacol. 2016;30(6):568-578. 


\section{Publish your work in this journal}

Neuropsychiatric Disease and Treatment is an international, peerreviewed journal of clinical therapeutics and pharmacology focusing on concise rapid reporting of clinical or pre-clinical studies on a range of neuropsychiatric and neurological disorders. This journal is indexed on PubMed Central, the 'PsycINFO' database and CAS,

and is the official journal of The International Neuropsychiatric Association (INA). The manuscript management system is completely online and includes a very quick and fair peer-review system, which is all easy to use. Visit http://www.dovepress.com/testimonials.php to read real quotes from published authors.

Submit your manuscript here: http://www.dovepress.com/neuropsychiatric-disease-and-treatment-journal 